\title{
Reflexiones sobre Redes de Conocimiento y Educación a Distancia en el Contexto Venezolano
}

\section{Reflections on Knowledge Networks and Distance Education in the Venezuelan context}

\author{
José L. López G*
}

\section{RESUMEN}

El presente artículo pretende hacer una discusión de las redes de conocimiento y de la educación a distancia, dando origen a una serie de consideraciones teóricas y a la indagación practica en el contexto venezolano. Se contextualiza este artículo en la concepción de la educación a distancia en toda su extensión. Autores como Albornoz y Alfaraz (2016), Castells (1994, 1996, 2000), Ibáñez y Serrano (1986), entre otros fundamentan este artículo. La metodología en la cual se encuentra enmarcada es una investigación de tipo documental con un diseño bibliográfico, Entre los elementos sinérgicos y conceptuales que se utilizan para el análisis se encuentra la definición de redes, la cual se aborda como una construcción simbólica o metafórica de la organización social, una construcción analítica y sintética de las estructuras de las relaciones e interacciones humanas bajo una construcción operativa y funcional de los sistemas de comunicación e información. Bajo esta perspectiva crítica se reflexionó sobre la praxis de la educación a distancia contribuyendo de esta manera a la construcción teórica y referencial relacionada con las tendencias en educación a distancia y desafíos sociales de las TIC en su dimensión ontológica.

Palabras clave: Educación a Distancia, Redes de Conocimiento, Contexto Venezolano

RECIBIDO: Marzo 2018

ACEPTADO: Junio 2018

\section{ABSTRACT}

This article aims to make a discussion of knowledge networks and distance education, giving rise to a series of theoretical considerations and to the practical investigation in

\footnotetext{
* Candidato a Doctor en Ciencias Administrativas y Gerenciales. Magíster en Administración de Empresas. Mención: Finanzas. Licenciado en Administración Comercial. Profesor Asociado a Dedicación Exclusiva de La Universidad de Carabobo. Valencia, Venezuela. Correo Electrónico: linojose38@gmail.com/jlopez2@uc.edu.ve
} 
José L. López G

Telos Vol. 20, No. 3 (2018). 404-430

the Venezuelan context. This article is contextualized in the conception of distance education in its entirety. Authors such as Albornoz and Alfaraz (2016), Castells (1994, 1996, 2000), Ibáñez and Serrano (1986), among others, support this article. The methodology in which it is framed is a documentary research with a bibliographic design. Among the synergic and conceptual elements that are used for the analysis is the definition of networks, which is approached as a symbolic or metaphorical construction of the social organization, an analytical and synthetic construction of the structures of human relations and interactions under an operational and functional construction of communication and information systems. Under this critical perspective, reflection on the praxis of distance education, thus contributing to the theoretical and referential construction related to trends in distance education and social challenges of ICT in its ontological dimensión.

Keywords: Distance Education, Knowledge Networks, Venezuelan Context

\section{Introducción}

Reflexionar desde la perspectiva educativa indudablemente es reflexionar en redes de conocimiento. Es pensar en saberes que se comparten, que circulan, que se distribuyen social e históricamente. Por ello las instituciones educativas, concebida como bien público nos envía necesariamente a la metáfora de la red y con ello a la idea de intercambio entre sujetos e instituciones que van construyendo un gran tejido reticular que conforma la denominada malla de la red, a través de nuevas y diferentes formas de relacionamiento y de trabajo.

Más allá de su materialización, si se conforma de manera virtual o con mixturas, la red tal cual como se concibe seria el resultado de la construcción de vínculos basados en la horizontalidad y la colaboración, donde los integrantes interrelacionados, no se perciben como amenaza, sino como aquellos que van a ayudar a crecer, a sostener y a fortalecer la vida de cada institución.

Faloh (2002) afirma que: "siempre que se trate de actividades humanas, el conocimiento es la base de cualquier tipo de red y por tanto todos los tipos posibles de redes humanas, en esencia son redes de conocimiento". El trabajo en red es trascendental para la creación de nuevos conocimientos. Lladó (2004) plantea "que 


\section{Reflexiones sobre Redes de Conocimiento y Educación a Distancia en el Contexto Venezolano}

solo facilitando el intercambio de conocimiento (...) será posible que la toma de decisiones, sobre aspectos que afectan a la gestión (...) sea más efectiva".

Las redes de conocimiento se plantean en el marco de la cibercultura, estos nuevos modos de ser, de comunicarse y de conocer lo que se va conformando en los nuevos ambientes sociales propiciado con las tecnologías de información y comunicación, creando nuevos ambientes que posibilitan construir nuevas relaciones sociales y nuevas maneras de compartir y recrear conocimientos.

Las instituciones educativas, deben ofrecer a la comunidad una formación flexible adecuada a sus necesidades, para ello, es fundamental el establecimiento de cambios en lo educativo que incorporen el uso de la tecnología. A su vez, la creación de nuevos espacios de formación exige al colectivo educativo la adquisición de competencias, habilidades, conocimientos y actitudes, con el objeto de aplicar responsablemente estrategias que permitan la gestión a distancia de los procesos académicos por medio de las TIC.

El compromiso de la educación, está en redefinir sus acciones para dar respuesta a las funciones que tiene encomendada con la sociedad, el conocimiento y el desarrollo, abrir espacios cónsonos con el desarrollo tecnológico para responder a las demandas de los entes sociales y a quienes tienen la responsabilidad de dirigir y desempeñar funciones técnicas y profesionales en las organizaciones.

Las redes académicas y científicas según Lopera (2000) están integradas por personas que conforman equipos de estudio y de trabajo para compartir informaciones, experiencias, documentación y diversos recursos; con el fin de lograr objetivos específicos en algún área del conocimiento. Se basan en el trabajo cooperativo, con altos niveles de participación entre pares académicos de una o varias instituciones, en ellas se desarrollan un aspecto determinante en el trabajo científico: el intercambio crítico de conocimientos y experiencias.Es así, como la educación a distancia, concebida como una modalidad educativa sustentada en el proceso de orientación-aprendizaje, es una ventana abierta a las demandas de la nueva sociedad 
José L. López G

Telos Vol. 20, No. 3 (2018). 404-430

del siglo XXI caracterizada por el desarrollo de las TIC y la globalización del mercado, las potencialidades que estas tecnologías aportan a los objetivos de los diseños instruccionales y de los fines planteados en el proceso de enseñanza en la modalidad de educación a distancia y las redes, con el acceso de los estudiantes a la información, de manera responsable, en libertad, autónoma e interactivamente para generar conocimientos y transmitirlos, colocándolos en una situación privilegiada de ser los constructores de su propio conocimiento.

De acuerdo con lo anterior, el presente artículo, se centra en realizar una discusión sobre las redes de conocimiento y la educación a distancia en el contexto venezolano, visualizando posibilidades de cambios y redefiniciones de la educación a distancia, a partir de la incorporación planificada de redes de conocimiento estratégicas y herramientas tecnológicas dispuestas en Internet.

\section{Método de Investigación}

El presente artículo, plantea que el análisis crítico del estudio, sigue una metodología que permite considerarla como una investigación de tipo documental con un diseño bibliográfico. Según los autores Palella y Martins (2010) se fundamenta el diseño bibliográfico en:

"La revisión sistemática, rigurosa y profunda del material documental de cualquier clase. Se procura el análisis de los fenómenos o el establecimiento de la relación entre dos o más variables. Cuando opta por este tipo de estudio, el investigador utiliza documentos, los recolecta, selecciona, analiza y presenta resultados coherentes. (p.87)

Derivándose una estructura teórico-conceptual, que permitirá establecer las relaciones y tendencias que configuran las redes de conocimiento y la educación a distancia en el contexto venezolano. 
Reflexiones sobre Redes de Conocimiento y Educación a Distancia en el Contexto Venezolano

\section{La Sociedad en Red}

La socialización del hombre junto con sus experiencias y acciones, le ha permitido percibir nuevos escenarios y tomar decisiones con mayor seguridad en cada oportunidad. La base de esa seguridad está dada por la posibilidad de utilizar el conocimiento acumulado.

El concepto de red (del latín rete) ha adquirido diversas acepciones registradas durante más de tres siglos, del XVI al XIX, en diferentes campos de conocimiento, como la biología, la geografía, la medicina y la ingeniería. Después de la segunda guerra mundial, el concepto de red adquirió nuevas connotaciones científicas, técnicas y sociales desde la perspectiva de la antropología, la economía, la psicología y la sociología, que han contribuido a la construcción de conceptos disciplinarios e interdisciplinarios.

Según Albornoz y Alfaraz (2016):

"En ciertas fuentes, el término "red" está asociado difusamente al concepto de sistemas, haciendo referencia a una totalidad organizativa compuesta por partes relacionadas. Desde esta perspectiva, las redes son vistas como una forma distribuida de acumular masa crítica. En otros contextos teóricos más recientes, las redes son constitutivas de los procesos de innovación, bajo una mirada que enfatiza la heterogeneidad de los actores involucrados. En otros contextos, más próximos al desarrollo de las tecnologías de información y comunicación, el término "red" adquiere un sentido que lo identifica preponderantemente con la web". (p. 131).

Wellman y Hampton (1999) sostienen que el patrón de relaciones en una red es sustancialmente el mismo que su contenido, en síntesis, son el tipo de representaciones gráficas y de análisis respectivos con una descripción cuantitativa y estadística de la densidad y distribución de las relaciones de los miembros de una red; sólo las investigaciones que se ocupan de la historia, el contexto y el contenido de las relaciones aportan conocimientos significativos sobre las formas de organización social, la sociabilidad, la cohesión social, el control y el poder.

En este sentido, Mercklé (2004) plantea que la noción de red comporta una dimensión metafórica, una topológica y una organización que corresponde a las 
José L. López G

Telos Vol. 20, No. 3 (2018). 404-430

relaciones y atributos de las relaciones entre individuos que contribuyen a modelar las estructuras sociales. La relevancia social de cualquier unidad social está condicionada por su presencia o ausencia en las redes específicas. La ausencia en la red dominante lleva a una estructura irrelevante.

Según Castells (1994):

"Estar desconectado de la red es equivalente a no existir en la economía global. La posición en la red, es decir, la función obtenida en la nueva división internacional del trabajo, se convierte en un elemento esencial para definir las condiciones materiales de existencia de cada país o región" (p. 39-40).

En base a los distintos puntos de vista, se plantea que la red representa una construcción simbólica o metafórica de la organización social; una construcción analítica y sintética de las estructuras de las relaciones e interacciones humanas; y una construcción operativa y funcional de los sistemas de comunicación e información. Tobón (2015) plantea que la sociedad en red, son aquellas comunidades que se dedican a interconectar información por medios tecnológicos, la cual se procesará, almacenará y transmitirá sin inconvenientes de tiempo, distancia, volumen.

Castells (2000) la define de la siguiente manera:

"Esa sociedad red es la sociedad que yo analizo como una sociedad cuya estructura social está construida en torno a redes de información a partir de la tecnología de información microelectrónica estructurada en Internet. Pero Internet en ese sentido no es simplemente una tecnología; es el medio de comunicación que constituye la forma organizativa de nuestras sociedades, es el equivalente a lo que fue la factoría en la era industrial o la gran corporación en la era industrial. Internet es el corazón de un nuevo paradigma socio técnico que constituye en realidad la base material de nuestras vidas y de nuestras formas de relación, de trabajo y de comunicación. Lo que hace Internet es procesar la virtualidad y transformarla en nuestra realidad, constituyendo la sociedad red, que es la sociedad en que vivimos". (p.18)

La sociedad en red, expresa los procesos, intereses, valores y las instituciones sociales. Específicamente constituye la base material y tecnológica de la sociedad red, la infraestructura tecnológica y el medio organizativo permite el desarrollo de una serie de nuevas formas de relación social que no tienen su origen Internet, que son 


\section{Reflexiones sobre Redes de Conocimiento y Educación a Distancia en el Contexto Venezolano}

fruto de una serie de cambios históricos pero que no podrían desarrollarse sin Internet. Esa sociedad red es la sociedad que se analiza como una sociedad cuya estructura social está construida en torno a redes de información a partir de la tecnología de información estructurada en Internet, la cual no es simplemente una tecnología; es el medio de comunicación que constituye la forma organizativa de nuestras sociedades, es el equivalente a lo que fueron las organizaciones en la denominada era industrial o la gran corporación en la era industrial.

La sociedad en red es una arista que deja de ser transmisora de información y que, junto con la formación del capital humano, y sin abandonar su función formativa, comienza a ser la administradora del conocimiento, basada en la información y por medio de la lógica investigativa, que el colectivo produce. Las instituciones educativas son aquellas las cuales se reelabora y genera conocimiento, y esto es algo que la sociedad en su conjunto requiere para su desarrollo armónico y evolutivo del sector educativo de la sociedad como gestor del conocimiento. El desempeño de estas nuevas funciones exige de las instituciones de educación una filosofía y una estructura administrativa diferentes de las convencionales, centradas en las personas mucho más que en el cumplimiento de objetivos.

\section{Las Tecnologías de Información y Comunicación}

Las tecnologías de la información y comunicación (TIC) han experimentado en los últimos años un espectacular desarrollo, difundiéndose en la mayoría de la población mundial, el consumo de Internet, el cual es un ítem que se utiliza frecuentemente como indicador de este fenómeno y según We Are Social (2017) más de 3.750 millones de personas se encuentran online; de los cuales el 50\% del tráfico web procede de dispositivos móviles, con un crecimiento de nada menos que el $30 \%$ respecto al año anterior. El desarrollo de las Tecnologías de Información y Comunicación (TIC) han generado una serie de ventajas en relación con la información y su acceso, estos cambios han producidos en las últimas décadas impactos de forma significativa en la Educación. 
José L. López G

Telos Vol. 20, No. 3 (2018). 404-430

Correa y Gómez (2009), citando a la Asociación Americana de las Tecnologías de la Información (Information Technology Association of America, ITAA), definen a las tecnologías de información y comunicación como:

"El estudio, diseño, desarrollo, implementación, soporte y administración de los sistemas de información basados en computadoras, en particular sus aplicaciones de software y hardware, así como el uso de las computadoras electrónicas y sus programas para la conversión, almacenamiento, procesamiento, transmisión y seguridad sumados a los medios de comunicación que ofrecen" (p. 39).

En cambio, de manera más amplia, la Organización de las Naciones Unidas (2002) parte de la definición de informática:

"ciencia que trata el diseño, realización, evaluación, uso y mantenimiento de sistemas que procesan información; incluyendo hardware, software, aspectos organizacionales y humanos, así como sus implicaciones industriales, comerciales, gubernamentales y políticas, concibe las tecnologías de la información como las aplicaciones tecnológicas (artefactos) de la informática en la sociedad, y las TIC como la combinación de las tecnologías de la información con otras tecnologías relacionadas, específicamente las tecnologías de las comunicaciones" (p. 12 -13).

Las TIC son aquellas tecnologías que permiten la adquisición, almacenamiento, procesamiento, evaluación, transmisión, distribución y difusión de la información, dichas TIC son desarrolladas mediante la convergencia de la informática, las telecomunicaciones, la electrónica y la microelectrónica, las TIC constituyen un nuevo sistema tecnológico con un amplio campo de aplicación. Castells (1996) considera que los rasgos más relevantes de las TIC son:

- Tecnologías para actuar sobre la información, no sólo información para actuar sobre la tecnología.

- Tienen gran capacidad de penetración y efecto en la economía, pues la información es parte integral de toda actividad humana, tanto individual como colectiva.

- La capacidad y la lógica de interconexión que todo sistema de TI utiliza permiten una interconexión rápida y barata. 
- Permiten el reprogramar y re-equipar a las organizaciones.

- Convergencia de tecnologías específicas para el desarrollo de estas.

De igual manera, Castells y Himanen (2002) reiteran que el paradigma de las nuevas tecnologías se basa en tres características: la capacidad de procesamiento de las TIC- en términos de volumen, complejidad y velocidad-, la habilidad de recombinarse y la flexibilidad de distribución. Es importante destacar que las TIC se caracterizan por tener aplicación en diferentes facetas de la actividad humana, afectando la forma de generación de información y de conocimiento, la forma de relacionarse entre sí de los individuos y la relación con las instituciones educativas.

En este sentido, por ejemplo, las universidades han rediseñado políticas para obtener los mejores resultados dentro de la institución, adaptándose a los nuevos paradigmas de gestión de la información y la comunicación; todo esto con la finalidad de tener una mejor relación con la población universitaria, y mayor proyección como ente transformador de la sociedad.

Joyanes (2001) señala que las TIC juega un papel importante para la sociedad, ya que tiene un valor social y económico y que representa el progreso tecnológico para las organizaciones universitarias. El impacto que han tenido las Tecnologías de Información y Comunicación (TIC) en la transformación de los modos de hacer y de las prácticas cotidianas ha sido extraordinario.

Según Peña y Gardié (2011):

En el ámbito específico de las TIC, éstas han venido transformando la realidad social, situación que está a la vista, pues han permeado todos los ámbitos de la vida: familiar, el educativo, el laboral, el comunitario, el político y hasta el privado. La ola de despliegue tecnológico, cada vez más sofisticada a partir de finales del siglo XX y comienzos del XXI, ha simplificado muchas actividades que antes eran lentas, engorrosas o de elevados costos. El individuo, por su parte, ha podido enriquecer sus saberes sobre la realidad local, regional, nacional e internacional que lo circunda (p. 99).

Asimismo, Majó y Pere (2003) afirman que: 
José L. López G

Telos Vol. 20, No. 3 (2018). 404-430

"La escuela y el sistema educativo no solamente tienen que enseñar las nuevas tecnologías, no sólo tienen que seguir enseñando materias a través de las nuevas tecnologías, sino que estas nuevas tecnologías aparte de producir unos cambios en la escuela producen un cambio en el entorno y, como la escuela lo que pretende es preparar a la gente para este entorno, si éste cambia, la actividad de la escuela tiene que cambiar" (p.35).

Caro (2015) plantea que las nuevas tendencias tecnológicas en la educación están cambiando la forma de relacionarse los estudiantes entre sí, con sus profesores e incluso con la institución, ya que la formación es más flexible, personalizada y de múltiple soporte, como, por ejemplo, adaptada a cada una de las necesidades del estudiante, sus competencias, el uso de internet y la telefonía móvil.

Es de resaltar que las universidades que están adoptando el uso de las TIC, han detectado la necesidad de dotar a las distintas Facultades, de una serie de infraestructuras tecnológicas apropiadas para el cambio educativo, y de desarrollar planes de formación del profesorado para el uso de estas nuevas Tecnologías de la Información y la Comunicación (TIC) y de proporcionar ayuda para que pueda acceder a ellas un mayor porcentaje de población.

Cabero (2007) plantea que con la incorporación de las TIC, se debe considerar que las universidades del país tienen un gran desafío al asumir las nuevas tendencias e incorporarlas en sus ambientes tanto presenciales como virtuales, de manera que puedan visualizarse todas las posibilidades de enseñanza, tales como la ruptura de las barreras espacio temporales entre docentes y estudiantes, los entornos de aprendizaje más flexibles e interactivos, así como el autoaprendizaje, el aprendizaje colaborativo, cooperativo, la formación permanente, entre otros.

En este sentido, resulta innegable que las funciones de la universidad, conforman una cadena de referencia indispensable para garantizar la misión, visión y valores de la universidad; sin embargo, se observan muchas limitaciones y en algunos casos hasta estancamiento en la administración de la función docencia, tímida participación en la función de extensión, limitada participación en la función investigación y escasa producción. 


\section{Reflexiones sobre Redes de Conocimiento y Educación a Distancia en el Contexto}

Venezolano

Niño (2017) señala aspectos a considerar como los altos costos de los equipos, el mantenimiento, la conectividad y los insumos, sin dejar de mencionar el bajo uso de computadores por parte del cuerpo docente y su falta de formación en el área, elementos que según el autor vienen dados por la resistencia al cambio o por las falencias de políticas de capacitación en las instituciones donde laboran.

Con relación a esto se puede considerar que las potencialidades de las TIC en la educación no alcanzan su máximo aprovechamiento ante elementos como la dotación, la formación y el acompañamiento de programas o políticas que fomenten su uso e incorporación formal en el proceso educativo.

Con estas ideas, es propicio llevar la reflexión a la universidad venezolana actual, ya que estos espacios educativos constituyen un modelo clásico de organización como lo establece el artículo 7 de la Ley de Universidades (1970), donde se distinguen las funciones básicas de enseñanza (docencia), investigación y extensión, áreas en las cuales los avances tecnológicos tienen pertinencia y son de gran aplicabilidad e importancia.

Según Duplass (1995) La universidad debería estar más preocupada por enseñar áreas del conocimiento con una perspectiva interactiva, de ambientes simulados y hasta de laboratorios automatizados. Las dificultades financieras, de espacios, de disponibilidad de equipo y de software, invitan a que las escuelas desarrollen cursos, programas o espacios más efectivos.

Desde esta perspectiva, en Venezuela se han realizado algunos esfuerzos gubernamentales a través del Ministerio del Poder Popular para Educación Universitaria, Ciencia y Tecnología, a partir de la incorporación de proyectos educativos y dotaciones tecnológicas en los últimos años, tal es el caso del Centro Nacional de Tecnologías de Información (2014) que promovió la distribución de las tabletas Canaima, a través del Proyecto Canaima Educativo, actividad impulsada para fomentar el uso de los sistemas operativos libres y contribuir con la independencia 
José L. López G

Telos Vol. 20, No. 3 (2018). 404-430

tecnológica como política pública del Estado y garantizar el acceso de la tecnología en los jóvenes universitarios en igualdad de condiciones.

Sin embargo, estos aspectos no son considerados eficientes, ya que se siguen presentando inconvenientes relacionados con él envió de recursos financieros, así como el software y hardware de las herramientas y su aplicación correcta en el área educativa; aspectos que también dependen por un lado de la aceptación y de la disposición de los usuarios para el uso la tecnología, y por otro, del compromiso de los directivos del ente gubernamental para incentivar su aplicación (CEPEP, 2009).

Así pues, el desarrollo de las nuevas tecnologías, las posibilidades de telecomunicaciones, la "amigabilidad" del software y las propuestas dentro de la psicología educativa así como de la psicología cognoscitiva (cognición situada) que han incidido en los espacios educativos, su estructura y hasta sus componentes (modelo educativo, modelo de enseñanza, modelo de aprendiz, etcétera), invitan a favorecer la creación de un nuevo paradigma en la enseñanza de las tecnologías del cómputo, así como a repensar los avances de la tecnología de la interconexión aplicada a la educación, y dirigida en gran medida hacia el diseño de redes de conocimiento.

Se trata de establecer con claridad el balance entre formación presencial y formación virtual. Además, las universidades han de analizar cuál será la demanda (local, regional, nacional o multinacional) de los nuevos servicios, qué perfiles tendrán sus estudiantes y qué tipo de formación pretende dar. Esta visión debería partir de un análisis de las potencialidades de estas tecnologías, no sólo para saber qué se puede hacer con ellas sino, fundamentalmente, para decidir qué se quiere hacer con ellas. Las TIC pueden ampliar el alcance y el grado de diversificación de la oferta formativa de las universidades, dirigida a nuevos colectivos de estudiantes: estudiantes a tiempo parciales, estudiantes que siguen sus estudios desde sus hogares, formación continua en el lugar de trabajo, etc. Las universidades pueden convertirse, de este modo, en instituciones que suministran formas múltiples de aprendizaje. 


\section{Educación a Distancia}

La incorporación de las TIC a nivel de la educación universitaria venezolana se remonta al año 1997, como recurso de apoyo en la modalidad de estudio presencial o en otros casos como recurso didáctico en ambientes de estudios semipresenciales. Curci (2004) establece que la entrada de las TIC en las universidades venezolanas se ha conseguido a través de las siguientes etapas:

- Sensibilización al cambio

- Sensibilización en el uso de herramientas TIC

- Capacitación

- Diseño y producción de contenidos

- Dictado de cursos

- Interés de las autoridades

- Proyecto formal de enseñanza virtual.

Ibáñez y Serrano (1986) definen la Educación a Distancia:

"La enseñanza a distancia es un sistema multimedia de comunicación bidireccional con el alumno alejado del centro docente, y facilitado por una organización de apoyo, para atender de un modo flexible el aprendizaje independiente de una población, masiva, dispersa. Este sistema suele configurarse con diseños tecnológicos que permiten economías de escala." ( $p$ 939-953)

Según Romero y Tobón (2000) la modalidad Educación a Distancia se define como:

"Una desincronía en el espacio básicamente la enseñanza, es decir, el instructor y el aprendiz no están físicamente en el mismo lugar. Generalmente la Educación a Distancia es no presencial y actualmente la mayoría se desarrolla con base en procesos ON LINE. Frecuentemente la Educación a Distancia no se da tampoco en el mismo momento (desincronía temporal), por ejemplo, si se emplea la correspondencia o el correo electrónico o el Web, pero bien puede darse en el mismo momento si se emplean medios como la televisión (interactiva o no) o los grupos de discusión ("chat") electrónicos con el Internet" (p. 107-116). 
José L. López G

Telos Vol. 20, No. 3 (2018). 404-430

Obsérvese que, a estas definiciones, intencionalmente lejanas en el tiempo, para adaptarlas a los nuevos tiempos, sólo habría que agregarles, en algunos casos, la referencia a los medios electrónicos y tecnologías interactivas que pueden permitir, incluso la enseñanza a distancia cara a cara (a través de videoconferencia) y el aprendizaje en grupos virtuales.

Las demandas de la sociedad del conocimiento en relación con la formación virtual hacen confluir un conjunto de disciplinas -tecnológicas, pedagógicas, políticas, etc.- que deben coordinarse para ofrecer una respuesta estructurada y relevante en el marco de diferentes contextos educativos - universitarios con lo cual cobra pertinencia la educación a distancia. La finalidad la cual debe estar orientada la educación a distancia es la enseñanza de procedimientos de regulación autónoma por parte del estudiante, tal independencia no involucra alimentar el individualismo ni el egoísmo, muy por el contrario, el que funge de tutor debe procurar el intercambio a través del uso de las TIC, sin abusar de ella.

En atención a esto último, cobra importancia lo planteado Casas (1987) quien supone que deben aplicarse las teorías y metodología requerida por la educación a distancia, tales como la planificación estratégica y operacional, así como la conformación de tecnologías organizacionales y educativas disponibles en la Universidad.

La modalidad de Educación a Distancia al ser propugnada en un sistema tecnológico que integre las competencias que se aspiran alcancen los participantes con la de la comunicación, asegura un tipo de información bidireccional, sustituyendo, de esta manera, la direccionalidad personal en el aula por parte del profesor hacia el alumno, por la acción sistemática y conjunta de diversos recursos didácticos a través del apoyo de tutorías. Tal procedimiento, propicia el aprendizaje independiente o auto aprendizaje en los estudiantes, pero con la exigencia académica y los criterios de calidad establecidos desde la Universidad, como manera de favorecer el éxito 


\section{Reflexiones sobre Redes de Conocimiento y Educación a Distancia en el Contexto Venezolano}

profesional de los egresados en correspondencia con el perfil profesional que se haya establecido.

\section{Redes de Conocimiento en el Contexto Venezolano}

La socialización del conocimiento implica su propagación en todos los sentidos, no se trata de que sólo a partir de los centros de investigación, instituciones educativas o cualquier insistencia de poder así legitimado se difunda. Las redes de conocimiento han sido definidas por diversos autores, siempre con características y objetivos generales similares, las variaciones son muy leves y están en dependencia de las particularidades y necesidades del contexto donde se originan.

Lopera (2000) define a la red de conocimiento como:

"un grupo multidisciplinario de personas e instituciones que se asocian para investigar o desarrollar proyectos con sentido social. Su finalidad es mejorar la calidad del trabajo académico y científico, crear y fortalecer la cooperación y el aprovechamiento de recursos y posibilitar el libre flujo de información entre los grupos sociales. Esto solo es posible si los actores se fundamentan en principios como la solidaridad y la integración constructiva, creativa, es decir, al mismo tiempo que se afronta una globalización de la información, se proponga y construya una globalización de la solidaridad" (p. 29).

Las redes de conocimiento son un mecanismo de intercambio social que relaciona a diferentes organizaciones o individuos las cuales se utilizan para promover el intercambio de información, compartir metodologías y prácticas de trabajo, colaborar en iniciativas tales como la capacitación, investigación y desarrollo y acumular conocimiento basado en las complementariedades, la reciprocidad y el intercambio.

Artiles (2002) propone dos visiones diferentes para definir las redes de conocimiento, plantea que para un investigador es un:

"agrupamiento comunicacional y participativo entre un grupo de individuos que intercambian información, conocimientos, ideas y experiencias; cuyas relaciones se establecen con miras a cooperar para un fin común, ya sea un proyecto de investigación, un tema de trabajo, la preparación de una actividad docente, la cooperación y desarrollo". (p.29) 
José L. López G

Telos Vol. 20, No. 3 (2018). 404-430

Por otra parte, establece que para un documentalista (bibliotecario, especialista en información, archivólogo, etcétera) es un:

"agrupamiento de personase instituciones dedicadas a la producción y difusión de la información sobre un tema determinado, cuyo énfasis es la información y que facilita prestar servicios al conjunto de usuarios interesados en un área del conocimiento determinada". (p. 30)

Al respecto el concepto que maneja Moreno (2005), sobre redes de conocimiento citando a Cónsoli y Molina:

“...está asociado a por lo menos dos aspectos centrales: el primero, que hace referencia al elemento humano y su vinculación en el estrato social, y el segundo, a las dinámicas inherentes a los patrones de organización que adoptan los mismos en función del contexto con el que interactúan”. (Pp.18-19)

El concepto de red abandona la concepción casi genérica de elemento eminentemente técnico, para adoptar una manifestación ampliamente relacionada con el orden socio-organizacional económico, cultural, y el desarrollo de la cooperación interuniversitaria de la sociedad de la información y el conocimiento, en el que las instituciones de educación desarrollan su actividad docente y científica. Esta evolución pone de relieve una necesidad creciente a la hora de generar mecanismos de cooperación.

Sin lugar a dudas, las redes de conocimiento se ven fortalecidas por la extensión de las actuales tecnologías, que aportan posibilidades de colaboración hasta ahora no exploradas. Las TIC, como se ha dicho, facilitan la organización de cursos a nivel internacional con carácter interactivo, así como el intercambio de información entiempo real. La red, entendida como forma de colaboración no jerárquica entre las instituciones educativas o entre éstas, la Administración Pública y el sector productivo, supone una apuesta estratégica de cara al futuro desarrollo.

Según Moreno (2005) las dimensiones en que se desarrollan las redes de conocimiento pueden ser de distintos alcances, según los propósitos y los intereses en campos y objetos de estudio; así, tenemos:

- Personales, como trabajadores, estudiantes, profesores, investigadores. 
- Institucionales. Desde el salón de clases en una escuela tradicional o en la comunidad de aprendizaje de un ambiente virtual, hasta entre instituciones o países, se pueden compartir centros de documentación, servicios bibliotecarios y, en general, servicios de información amplia y especializada.

- Población en general, que así puede revalorar, sistematizar y legitimar el conocimiento cotidiano y establecer redes de colaboración para resolver problemas.

Muñoz, Rodríguez y Barrera (2013) plantean que existen una gran variedad de estrategias para vincularse con el trabajo en red, que pueden crearse de acuerdo con nuestras condiciones, propósitos, necesidades de conocer y creatividad; menciono las siguientes:

1. Se plantea a las instituciones académicas, gobierno y empresas como los elementos clave. Así, el gobierno regula y apoya, las instituciones académicas generan conocimiento y las empresas en su aplicación pueden generan conocimiento nuevo.

2. Al interior de las empresas en el desarrollo profesional, el conocimiento que llega de fuera y el generado dentro por sus instancias de investigación y desarrollo, se recupera de la creatividad y potencialidad de sus trabajadores, desarrollando y aplicando lo aprendido.

3. A través de las TIC se organizan redes, muchas veces informales, para tratar temas de interés mundial, como: "El patrimonio cultural de la humanidad", "Desarrollo y sostenibilidad", “Aprender en comunidad a vivir la diversidad", "Educación para la paz y la convivencia", "Cultura de género" y muchos más.

Muñoz, Rodríguez y Barrera (2013) comentan que entre las ventajas que se derivan de la colaboración en redes se destacan las siguientes: 
José L. López G

Telos Vol. 20, No. 3 (2018). 404-430

- Las redes otorgan preferencia a las estructuras más flexibles, que permiten extender y diversificar las actividades de cooperación, experimentarlas soluciones y compartir los riesgos.

- Las redes constituyen el mejor medio para construir una comunidad de acción, basada en el reconocimiento recíproco de la labor de los distintos colaboradores.

- La participación en redes incrementa el número de unidades organizativas universitarias afectadas por la colaboración.

- Las redes permiten organizar la cooperación interuniversitaria dentro y fuera de las fronteras del país de forma más equilibrada y eficaz.

Una de las funciones básicas que suele desarrollar las redes universitarias es la transmisión de información entre las instituciones de enseñanza superior, la Administración Pública y los sectores productivos, en su caso, el rápido intercambio de información viene facilitado por los avances tecnológicos e informáticos más recientes. La red puede difundir al resto de las instituciones educativas las experiencias positivas de cada una de ellas y puede contribuir a reducir el impacto de los aspectos más débiles de cada institución, en beneficio del conjunto.

La creación de redes es importante ya que concretan así colaboraciones destinadas a mejorar la calidad académica. En las redes pueden establecerse estándares para una calidad continuada y para un proceso sistemático de esfuerzo en favor de la misma. Es previsible que las instituciones educativas de mayor excelencia académica se comprometan en mecanismos de colaboración que preserven o superen los estándares de calidad conseguidos.

Otro elemento se refiere al hecho de que el sistema de red permite comunicar la investigación producida en las universidades con la que se efectúa en otros centros de investigación y laboratorios de empresas. Se consigue así una división del trabajo en proyectos comunes con finalidades compartidas, donde se diluye, en gran medida, la frontera entre la investigación básica y la investigación aplicada. 


\section{Reflexiones sobre Redes de Conocimiento y Educación a Distancia en el Contexto Venezolano}

Se subraya ante todo el tema del liderazgo y de la toma de decisiones. El proceso de toma de decisiones en las organizaciones universitarias implicadas en las redes se desarrolla a un doble nivel. Por un lado, el nivel central legitima la red en función de las estrategias de cada instituto, evalúa sus resultados, desempeña eventualmente los medios de apoyo y desarrollo y puede comprometer colaboraciones que afectan a los modos de gobierno de la propia institución. Las redes suelen iniciarse en torno a personas con la experiencia y el interés adecuados para llegar a acuerdos estables y duraderos. La motivación de todas las personas implicadas en la red suele ser decisiva a la hora de concretar los acuerdos de colaboración.

Pérez y Castañeda (2009) plantean que, para el desarrollo de las redes de conocimiento de la educación a distancia, esta debe presentar tres rasgos tendenciales más frecuentes como los que se mencionan a continuación:

1) Deben contribuir a la descentralización de la toma de decisiones políticas, financieras, administrativas y académicas en las instituciones, respetando las autonomías universitarias.

2) Promover a la integración de comunidades académicas y profesionales para compartir conocimientos y experiencias en los ámbitos local, regional y nacional;

3) Fortalecer la convergencia de sistemas educativos multisectoriales con instituciones educativas, gubernamentales y civiles o empresariales. Sin embargo, las nuevas formas de organización y funcionamiento de las redes de educación superior a distancia implican la ruptura o transformación de viejos paradigmas normativos y relaciones de poder político, económico, científico y tecnológico de las universidades.

Un requisito básico para el buen funcionamiento de la red es el establecimiento de relaciones a largo plazo. Esto implica una definición de los objetivos de la red en el marco del plan estratégico de cada instituto educativo. Las redes imponen la coordinación de objetivos e intereses, respetando la autonomía propia de cada 
José L. López $G$

Telos Vol. 20, No. 3 (2018). 404-430

institución y la participación de todos los colectivos implicados. A largo plazo, redes e institutos educativos han de administrarse dentro de una política global de desarrollo que permita la conexión entre dichas instituciones y las estrategias territoriales tanto delos sectores empresariales como de la administración local. Tampoco hay que olvidar la necesaria preservación de la identidad de las universidades. En toda estrategia los objetivos han de quedar claros y ser explícitamente comunicados; en este sentido, las redes han de ser compatibles con la misión y los valores básicos de la institución.

\section{Conclusiones}

La UNESCO (2005), plantea el reto de transformar la sociedad de la información en sociedades del conocimiento, haciendo énfasis en que la información debe insertarse en un proceso de aprendizaje y conocimiento compartido, por lo que se ha de convertir en formación real, en actividad, en participación. Las redes de conocimiento nacen principalmente de la producción y difusión de éste a través, generalmente, de los nuevos medios y/o recursos tecnológicos actuales, los institutos educativos, que constituye el modelo clásico de organización de educación, se distingue por tres funciones básicas: enseñanza, investigación y extensión.

En este modelo, el conocimiento se genera mediante la investigación, y son transferidos a individuos que los necesitan mediante la docencia y los cuales son aplicados a la sociedad por sus egresados o por los profesores a través de proyectos o servicios de asesoría hacia la comunidad. Ante tales circunstancias, es necesario aceptar que desde la educación básica hasta la superior se requieren programas emergentes para reorientar la acción docente, hacia aquella que vaya encaminada a la formación de personas calificadas y competitivas que protagonizaran los nuevos escenarios de la dinámica socio-económica, política y científico tecnológica nacional y mundial. 
Reflexiones sobre Redes de Conocimiento y Educación a Distancia en el Contexto Venezolano

Estos desafíos han sido abordados desde distintos documentos e instrumentos del Estado Venezolano, como en la Asamblea Nacional Constituyente (1999):

"El Estado reconocerá el interés público de la ciencia, la tecnología, el conocimiento, la innovación y sus aplicaciones y los servicios de información necesarios por ser instrumentos fundamentales para el desarrollo económico, social y político del país, así como para la seguridad y soberanía nacional...". (p.18)

Por otra parte, la Ley de Ciencia, Tecnología e Innovación, en los artículos del 11 al 19 pretende estimular la inversión en actividades de investigación y desarrollo en general, la formación de talento y el fortalecimiento de la demanda de Ciencia y Tecnología con el fin de incidir en la modernización y reactivación de este sector, incentivando la formación de redes empresariales, la vinculación con el sector académico y de investigación, así como el establecimiento de procesos de innovación que permitan una mejor inserción competitiva de la producción venezolana en los mercados nacionales, regionales y mundiales.

Para responder a las necesidades formativas derivadas de esta situación se debe potenciar la formación del profesorado, de todos los niveles educativos en general y de los universitarios en particular. La formación en redes de aprendizaje es un arma que se ha de convertir en la principal variable dependiente en todos los procesos de construcción, reconstrucción, crecimiento y mejora de la comunicación educativa. La complejidad del sistema de educación superior es mayor que cualquiera de los otros sistemas que conforman el sistema educativo de un país. Se pueden tener instituciones que son unifuncionales que ejercen fundamentalmente la docencia y otras multifuncionales que realizan las funciones de docencia, investigación y extensión.

$\mathrm{Al}$ respecto, Pereira y Suarez (2005) señalan que las universidades están llamadas a asumir un papel protagónico en los nuevos cambios, propiciando la investigación como eje fundamental, coincidiendo con otros autores como Avalos (2005) y Aguilera (2000) quienes además resaltan la innegable importancia de la educación y la 
José L. López G

Telos Vol. 20, No. 3 (2018). 404-430

investigación como ejes fundamentales de la política económica de los países desarrollados.

Bajo esta nueva perspectiva, la investigación debe tener la agilidad suficiente, para poder migrar entre una investigación básica de generación propia del saber y la otra investigación que es inmediata, que busca cómo resolver problemas en todos los ámbitos de la sociedad de conocimiento. Para lograr tal propósito, la universidad tiene como reto el empezar a innovarse al interior. Este llamado ha llevado muy especialmente a las universidades a constituirse en organizaciones capaces de analizarse, cuestionarse y transformarse a la luz de lograr una mejor comprensión y transformación de los problemas educacionales en concordancia a los nuevos retos de formación.

Estas transformaciones aún tendrán que franquear las barreras culturales que en algunos ámbitos educativos todavía prevalecen en cuanto al apego a la tradicional forma de concebir el proceso educativo subordinado a la enseñanza, según el cual el educador enseña y el alumno aprende. La efectividad de su aplicación dependerá de la superación de éste obstáculos y de la incorporación de métodos, técnicas, recursos y de la definición de postulados teóricos que respalden metodológicamente las prácticas pedagógicas y los principios del conocimiento de la educación a distancia mediada por TIC.

Las redes permitirán ver las necesidades desde nuevas perspectivas para poder innovar y dar solución a los problemas que se plantean. Éstas se están perfilando como espacio alternativo a la formación tradicional, promoviendo el aprendizaje y la reflexión sobre la propia experiencia, las redes permiten la creación de comunidades que facilitan el aprendizaje y crean un marco de intercambio de experiencias y vivencias.

Desde esta perspectiva, tenemos una serie de propuestas planteadas por Moreno (2005) relacionadas con la relación de personas que se organizan con el propósito de aprender, crear y compartir el conocimiento, entre las cuales se destacan: 
Reflexiones sobre Redes de Conocimiento y Educación a Distancia en el Contexto Venezolano

- Cerrar las brechas, al menos en lo que al conocimiento se refiere, con estrategias de vinculación para la cooperación y la colaboración, que hagan posible la movilidad del conocimiento que se genera, comparte aplica y recrea como factor fundamental para el desarrollo social.

- Canalizar los resultados de las investigaciones hacia la comunidad.

- Integración y funcionamiento de alianzas sólidas y atractivas para trabajar programas conjuntos de formación en todas las áreas y funciones educativas.

- Desarrollo de investigaciones conjuntas y compartidas.

- Colaboración en plataformas para la gestión del conocimiento, con criterios y estándares compartidos.

Las implicaciones e importancia del conocimiento en las universidades venezolanas, debe fundamentarse en la necesidad de prever el futuro desde una visión de interacción entre el conocimiento y el dominio de expertos para apoyar las comunidades de investigación, desarrollo e innovación I + D + I del sector educativo superior. Los importantes avances en desarrollo de tecnologías de información y comunicación (TIC), en la actualidad las universidades públicas autónomas han de superar las debilidades centradas en la capacidad de transferir conocimiento y posiciones institucionales ante la sociedad. La discusión de los puntos significativos discutidos revela el impacto de las TIC, centrada en los planteamientos del conocimiento constructivista y del aprendizaje significativo.

Paredes (2008) destaca que la producción del conocimiento en las universidades públicas autónomas venezolanas, difícilmente es considerado como un recurso estratégico de competitividad quizás por su escasa disponibilidad, accesibilidad o promoción y difusión; situación que genera, deficiencias para visionar posibles alternativas de solución ante situaciones presentadas en contextos internos y externos; así como, prever las implicaciones y resultados de decisiones institucionales.

Bricall (2000) plantea:

"Las redes suelen iniciarse en torno a personas con la experiencia y el interés adecuados para llegar a acuerdos estables y duraderos. La motivación de todas 
las personas implicadas en la red suele ser decisiva a la hora de concretar los acuerdos de colaboración; la masa crítica de personas involucradas en cada institución tiene que ser lo bastante importante para que los acuerdos no queden circunscritos a un mero interés individual. Un requisito básico para el buen funcionamiento de la red es el establecimiento de relaciones a largo plazo. Esto implica una definición de los objetivos de la red en el marco del plan estratégico de cada universidad. Las redes imponen la coordinación de objetivos e intereses, respetando la autonomía propia de cada institución y la participación de todos los colectivos implicados. A largo plazo, redes y universidades han de administrarse dentro de una política global de desarrollo que permita la conexión entre dichas instituciones y las estrategias territoriales tanto de los sectores empresariales como de la administración local. Tampoco hay que olvidar la necesaria preservación de la identidad de las universidades. En toda estrategia universitaria, los objetivos han de quedar claros y ser explícitamente comunicados; en este sentido, las redes han de ser compatibles con la misión y los valores básicos de la institución”. (p. 471)

En consecuencia, el uso de la planificación prospectiva ha de considerarse desde una perspectiva capaz de soportar el desarrollo permanente, la razón de ser y pertinencia de las universidades públicas ante la sociedad; por ello, es necesario iniciar un proceso de identificación de técnicas fundamentadas en el análisis de escenarios futuros o la participación de actores, a fin de relacionarlos con los componentes de gestión del sector universitario de manera de prever su interacción e integración, para lograr ventajas de desarrollo y consolidar estructuras que soporten la acción competitividad del sector. Se debe trabajar en condiciones idóneas, y entre ellas la fundamental es el reencuentro entre las instituciones educativas y la sociedad que las creó y a la que deben servir, es urgente que se avance hacia escuelas más vivas y sociedades más educativas.

\section{Referencias Bibliográficas}

Aguilera, Manuel (2000). La Investigación Universitaria: indispensable en los nuevos tiempos. Revista: Universitas, vol. 29, Ecuador (Pp. 11-12).

Albornoz, Mario y Alfaraz, Claudio (2016). La RICYT como práctica de trabajo en red. Redes de Conocimiento Construcción, Dinámica y Gestión. Editorial: Red Iberoamericana de Indicadores de Ciencia y Tecnología (RICYT). Argentina 
Reflexiones sobre Redes de Conocimiento y Educación a Distancia en el Contexto Venezolano

Artiles, Sara (2002). Las Redes del Conocimiento como Producto de la Gerencia de Información en Ambientes Académicos. II Edición de IBERGECYT (Gestión del Conocimiento: concepto aplicaciones y experiencias). La Habana Cuba.

Asamblea Nacional Constituyente (1999). Constitución de la República Bolivariana de Venezuela. Gaceta oficial 36.860. Venezuela

Avalos, Ignacio (2005). La investigación universitaria en tiempos de la sociedad del conocimiento. Revista Venezolana de Economía y Ciencias Sociales, Enero., vol.11, no.1, Venezuela (Pp.089-105).

Bricall, Jose (2000). Conferencia de Rectores de las Universidades españolas (CRUE), Informe Universidad 2000. Extraído de: https://www.oei.es/historico/oeivirt/cap9.pdfconsulta: 03/05/2018

Cabero, Julio (2007). Nuevas tecnologías aplicadas a la educación. Editorial McGraw-Hill. Uruguay.

Caro, Manuel (2015). Las 5 primeras tendencias de la educación 3.0. Extraído de: http://ideasqueinspiran.com/2015/05/07/principales-tendencias-de-laeducacion-3-0/consulta: 01-05-2018

Casas, Armengol (1987). Universidad sin clases: Educación en América Latina. Editorial Kapelusz. Venezuela.

Castells, Manuel (1994). Flujos, redes e identidades: una teoría crítica de la sociedad informacional. Flecha José, Freire Paulo, Giroux Henry, Macedo Donaldo, Willis Paul. Nuevas perspectivas críticas en educación. Editorial: Paidós Ibérica. España

Castells, Manuel (1996). La era de la Información. Economía, Sociedad y Cultura. Vol. 1. Editorial Alianza. España.

Castells, Manuel (2000). Internet y la sociedad red. Conferencia de Presentación del Programa de Doctorado sobre la Sociedad de la Información y el Conocimiento. Universidad Abierta de Cataluña, España

Castells, Manuel y Himanen, Pekka (2002). El Estado de bienestar y la Sociedad de la Información. El modelo finlandés. Editorial Alianza. España.

CEPEP (2009). El software libre en Venezuela y la Soberanía Tecnológica. Equipo Cooperativa Centro de Estudios para la Educación Popular. Extraído de: http://geminis.upel.edu.ve/comunidti/images/softwarLibre.pdf Consulta: 0205-2018.

Centro Nacional de Tecnologías de Información (2014). Proyecto Canaima Universitario. Extraído de: http://www.canaimaeducativo.gob.veConsulta: 03-05-2018

Correa, Alexander y Gómez, Rodrigo (2009). Tecnologías de la información en la cadena de suministro. Revista Dyna. Volumen. 76. No 157. España (Pp. $37-$ 48). 
José L. López G

Telos Vol. 20, No. 3 (2018). 404-430

Curci, Renata (2004). Diagnóstico de la educación superior virtual en Venezuela. En la educación superior virtual en América Latina y el Caribe. ANUIES-

UNESCO. Extraído

de:http://unesco.org.ve/dmdocuments/biblioteca/libros/EducVirtual_ALC.pdf consulta: 25-08-17.

Duplass, James (1995). Teaching software: Is the Supervised Laboratory Effective?.

Revista Computers in Education.Vol.24 No.4. USA (Pp.287-291).

Faloh, Rodolfo (2002). Redes del Conocimiento. Empresas de Clase en Cuba.II Edición de IBERGECYT (Gestión del Conocimiento: concepto, aplicaciones y experiencias). La Habana Cuba.

Ibáñez, Ricardo y Serrano, Gloria (1986). Pedagogía Social en la Universidad: Realidad y prospectiva. Universidad Nacional de Educación a Distancia. España

Joyanes, Luis (2001). Cibersociedad. Los retos sociales ante un nuevo mundo digital. Editorial Mc Graw-Hill. España.

Lopera, Hernando (2000). Integración de redes de conocimiento: una responsabilidad de la biblioteca universitaria. En $6^{\circ}$ Congreso Nacional de Bibliotecología y Documentación. Bogotá, Colombia

Lladó, Salamanca (2004). Redes Peer-To-Peer para la Gestión de Conocimiento en Destinos Turísticos. Extraído de: https://www.researchgate.net/profile/Esteve_Llado/publication/242098442_R edes Peer-To-

Peer_para_la_Gestion_de Conocimiento_en_Destinos_Turisticos/links/0dee c5245d16963566000000/Redes-Peer-To-Peer-para-la-Gestion-de-

Conocimiento-en-Destinos-Turisticos.pdf, consulta: 24-09-17.

Majó, Joan y Pere, Majo (2003). La Revolución educativa en la era Internet. Editorial: Ciss Praxis. España

Moreno, Manuel (2005). Redes de conocimiento en la educación a distancia. Revista: Apertura.Vol. 5, no 1. México (Pp. 7-23)

Mercklé, Pierre (2004). Sociologie des réseauxsociaux. Editorial: La Découverte. Francia

Muñoz, José; Rodríguez, David y Barrera, Aleix (2013). Herramientas para la mejora de las organizaciones educativas y su relación con el entorno. Revista: Perspectiva Educacional, Formación de Profesores, vol. 52, no 1. México (Pp. 97-123)

Niño, Ada (2017). Las TIC en la Educación Universitaria Venezolana: Una Reflexión desde la Perspectiva Docente. Revista Investigación Y Formación Pedagógica Revista Del Ciegc. No 6. Venezuela (Pp. 59-67) 
Organizacion de las Naciones Unidas (ONU) (2002). Information and Communication Technology in Education: Division of Higher Education. Francie

Palella, Santa y Martins, Feliberto (2010). Metodología De Investigación Cuantitativa. (Tercera edición). Editorial Fedeupel. Venezuela

Paredes, Ana (2008). Planeación prospectiva para las redes de conocimiento corporativo en las universidades públicas venezolanas. Revista: de Ciencias Sociales (RCS). Vol. XIV, No. 2. Venezuela (Pp. 274 - 285).

Pérez, Yudit y Castañeda, Malena (2009). Redes de conocimiento. Revista: Ciencias de la Información, vol. 40, núm. 1, enero-abril, Instituto de Información Científica y Tecnológica. Cuba (Pp. 3-20).

Pereira, Lilia y Suarez, Wendolin (2005). Estrategias para impulsar la investigación administrativa. Revista: Espacios. Vol. 26, No 1. Venezuela. (Pp.63-75).

Peña, Ana y Gardié, Gabriela (2011). Uso de las tecnologías de la información y comunicación (TIC) como herramienta didáctica en la especialidad de administración de la Universidad Nacional Experimental "Simón Rodríguez"(UNESR). Revista: Etic@ net, Vol. 9, No 11, Venezuela (Pp. 97-123)

Romero, Loaiza y Tobón, Lindo (2000). La universidad virtual: Análisis de la situación global. Revista Ciencias Humanas. Vol. 26, Colombia. (Pp.107116).

Sánchez, Jenny; González, Mayda y Sánchez, María (2012). La sociedad de la información: Génesis, iniciativas, concepto y su relación con las TIC. Revista UIS Ingenierías, vol. 11, no 1. Colombia (Pp. 113-128)

We Are Social (2017). Global overview. January. Extraído de: https://www. Slideshare. Net/wearesocialsg/digital-in-2017-global-overview. Consulta: 2509-17

Tobón, Sergio (2015). Sociedad del Conocimiento: Estudio documental desde una perspectiva humanista y compleja. Revista: Paradigma, vol. 36, no 2. Venezuela. (Pp. 7-36).

UNESCO (2005). Informe Mundial. Hacia las sociedades del conocimiento. Publicaciones Unesco. Francia.

Wellman, Barry y Hampton, Keith 1999). Living networked on and offline. Revista: Contemporary Sociology. Vol 28 No 6. USA. (Pp 648-654). 